\title{
Thermally regenerated fiber Bragg gratings in twin-air-hole microstructured fibers for high temperature pressure sensing
}

Tong Chen, Rongzhang Chen, Charles Jewart, Botao Zhang, John Canning, et al.

Tong Chen, Rongzhang Chen, Charles Jewart, Botao Zhang, John Canning, Kevin Cook, Kevin P. Chen, "Thermally regenerated fiber Bragg gratings in twin-air-hole microstructured fibers for high temperature pressure sensing," Proc. SPIE 8028, Fiber Optic Sensors and Applications VIII, 802807 (3 June 2011); doi: 10.1117/12.884994

Event: SPIE Defense, Security, and Sensing, 2011, Orlando, Florida, United States 


\title{
Thermally Regenerated Fiber Bragg-Grating in Twin-Air-Hole Microstructure Fiber for High Temperature Pressure Sensing
}

\author{
Tong Chen ${ }^{\mathrm{a}}$, Rongzhang Chen ${ }^{\mathrm{a}}$, Charles Jewart ${ }^{\mathrm{a}}$, Botao Zhang ${ }^{\mathrm{a}}$, John Canning ${ }^{\mathrm{b}}$, Kevin Cook $^{\mathrm{b}}$, Kevin P. Chen ${ }^{\mathrm{a}}$ \\ ${ }^{a}$ Department of Electrical Engineering, University of Pittsburgh, Pittsburgh, PA, USA 15261 \\ bInterdisciplinary Photonics Laboratories, School of Chemistry, University of Sydney, Sydney, Australia, NSW \\ 1430
}

\begin{abstract}
We present thermally regnenerated fiber Bragg grating in air-hole microstructured fibers for high temperature hydrostatic pressure sensing application. Saturated type I gratings were inscribed in hydrogen-loaded two-hole optical fibers using $248-\mathrm{nm} \mathrm{KrF}$ laser, and regenerated during annealing at $800^{\circ} \mathrm{C}$. The fiber Bragg grating resonance wavelength shift and peak splits were studied as a function of external hydrostatic pressure from 15 psi to 2400 psi. The grating pressure sensor shows stable and reproducible operation up to $800^{\circ} \mathrm{C}$. This paper demonstrates a multiplexible pressure sensor network technology for high temperature harsh environment using a single fiber feedthrough.
\end{abstract}

Keywords: Fiber Bragg grating, high temperature sensor, pressure sensor, regenerated grating

\section{INTRODUCTION}

Hydrostatic pressure measurements for high temperature environments are uniquely demanding and challenging for a wide range of applications in energy industry. Fast, accurate and reliable interrogation of gas pressure information ensures safe and efficient operations of gas turbine, coal boilers, power plants, and etc, where the operating temperatures range from $400^{\circ} \mathrm{C}$ to more than $1000^{\circ} \mathrm{C}$. Fiber optical sensors have always been considered as good candidates for harsh environment applications. In the past few years, high temperature pressure sensors based on Fabry-Peròt interferometry (FPI) have been successfully demonstrated [1-3]. On the other hand, fiber Bragg-grating (FBG) based sensors, in which Bragg wavelength shifts are interrogated with respect to environmental changes, have small in-line loss, good sensing resolution and wavelength and temporal multiplexing capability compared with FPI type sensors. Hundrends of type-I FBGs with same or different Bragg wavelength can be inscribed into a single fiber feedthrough to perform distributive sensing measurements. Different sensing parameters, such as temperature, strain, pressure and refractive index can be discriminated with advanced grating sensor designs. Therefore, FBGs are considered more suitable for multipoint sensing network applications. However, type-I gratings written in photosensitive fiber with UV radiation gradually lose their strength with the rise in temperature. Conventional FBGs can be erased from optical fibers and lost their functionalities at temperature above $500^{\circ} \mathrm{C}$ for short term or above $300^{\circ} \mathrm{C}$ for long term applications. The poor thermal stability seriously limited the implementations of FBG sensors in high temperature applications.

There have been lots of efforts in elevate the survival temperature of FBGs. It may involve different technical procedures in changing the chemical composition of fiber glass [4], Nitrogen-doping [5], fiber photosensitization [6], and also permanent damage grating written including type-II grating with ultrafast laser [7] and type-IIA grating with IR laser [8]. Recently, a new type of high temperature FBG was demonstrated, where grating structure is regenerated through annealing at high temperature, after the type-I seeding grating inscribed with UV radiation was erased [9-12]. The regenerated grating can operate at temperatures as high as $1295^{\circ} \mathrm{C}$ [11], which is higher than the operating temperature demonstrated with ultrafast laser written type-II gratings [7], and approaches the melting temperature of fiber glass. By carefully tuning the fiber doping, seeding grating strength, and annealing schedule, relatively strong high-temperature gratings with $\sim 35 \%$ reflectivity can be manufactured with well-defined spectral shape, narrow linewidth and low in-line loss [12]. The ultrahigh temperature stability, good grating qualities and relatively simple procedures make the regenerated gratings technique attractive to multiplexible high-temperature sensing applications.

*kchen@engr.pitt.edu; phone: 1-412-624-9675; fax: 412-624-8003

Fiber Optic Sensors and Applications VIII, edited by Stephen J. Mihailov, Henry H. Du, Gary Pickrell,

Anbo Wang, Alexis Mendez, Eric Udd, Proc. of SPIE Vol. 8028, 802807 · @ 2011 SPIE

CCC code: $0277-786 X / 11 / \$ 18 \cdot$ doi: $10.1117 / 12.884994$ 
The invention and rapid development of microstructure fiber in the last 15 years revolutionized a wide range of scientific and engineering research fields. The wide flexibilities in the geometry and optical properties of microstructure fibers, provide numerous opportunities in both novel designs and performance enhancements to fiber sensors. Traditional FBG sensor made in solid silica fibers shows good sensitivity in measuring axial strains. But when transverse stress such as directional force or hydrostatic pressure is applied to the fiber, the sensor response is very limited. Moreover, by merely measuring the Bragg wavelength shift, it is difficult to discriminate the temperature influence on the FBG sensor from transverse stress responses. Small cross-sensitivity and high transverse stress resolution cannot be achieved simultaneously [13]. This problem can be overcome by making fiber sensors using side air-holes microstructure fibers [14-17]. When a directional force or hydrostatic pressure is applied to the air-hole fiber, the deformation of air holes transfers the external perturbation into internal stress in the fiber core. The induced internal stresses in $\mathrm{x}$ and $\mathrm{y}$ transverse directions are different due to the side-hole geometry, and this external stress dependent birefringence can be interrogated through the separation of FBG resonant peaks in $\mathrm{x}$ and y polarizations. The transverse stress and pressure response of air-hole microstructure fibers can be numerically simulated with Finite element analysis (FEA) model. New fiber geometries can be designed with optimized size, sharp and positions for fiber core and air-holes, to achieve better transverse stress and pressure response and lower cross-sensitivities [18-19].

Previously, we demonstrated a high temperature pressure sensors inscribed in air-hole microstructure fiber with an ultrafast laser [20]. The type-II FBG shows stable and reproducible sensing operation over $800^{\circ} \mathrm{C}$. But the grating linewidth and strong laser induced birefringence need to be further optimized to allow better pressure sensing range and accuracy. The relatively large in-line loss with the type-II permanent damage also makes sensor multiplexing more difficult.

In this letter, we apply thermal regeneration technique to air-hole microstructure fiber gratings, and present a multiplexible high-temperature pressure FBG sensor. The regenerated grating is stable at $800^{\circ} \mathrm{C}$, and presents ultrasharp linewidth and minimum intrinsic birefringence. Hydrostatic gas pressure from 15 to 2400 psi is interrogated with the regenerated grating at $800^{\circ} \mathrm{C}$, and the sensing resolution can reach 15 psi with a 1-pm resolution tunable laser. The temperature and pressure responses can be differentiated with a 2x2 response matrix. Multiplexed regenerated grating sensors in a single fiber can serve as a single device for multipoint and simultaneous measurement of wide ranges of temperature and pressure, which provides a simple and reliable sensing solution in high temperature harsh environment.

\section{EXPERIMENTS}

Air-hole fibers used in this work have cladding diameter of $220-\mu \mathrm{m}$. Two large air holes with $90-\mu \mathrm{m}$ diameter are placed 20 - $\mu \mathrm{m}$ apart in $\pm \mathrm{x}$ direction. The fiber core is $9.7-\mu \mathrm{m} \times 7.5-\mu \mathrm{m}$ in size and $4.5-\mu \mathrm{m}$ away from the geometric center of the fiber. The microscopic cross-section image of the fiber is shown in the inlet of Fig. 1a, where the fiber core and cladding are illuminated air holes and surrounding. A piece of $20-\mathrm{cm}$ long air-hole fiber was stripped, end-cleaved and fusion spliced at both end with 1550-nm telecom single-mode fiber (Corning SMF-28). The telecom fiber has smaller cladding diameter of $125-\mu \mathrm{m}$. In order to seal the air-holes from ambient pressure, multiple re-splices are needed after the initial splice, as shown in fig. 1b. The transmission power is monitored during fusion splice to ensure best optical coupling and splicing strength. Less than $1 \mathrm{~dB}$ loss is achieved with the splice, and the fiber is proved durable under hydrostatic pressure up to $2400 \mathrm{psi}$. 

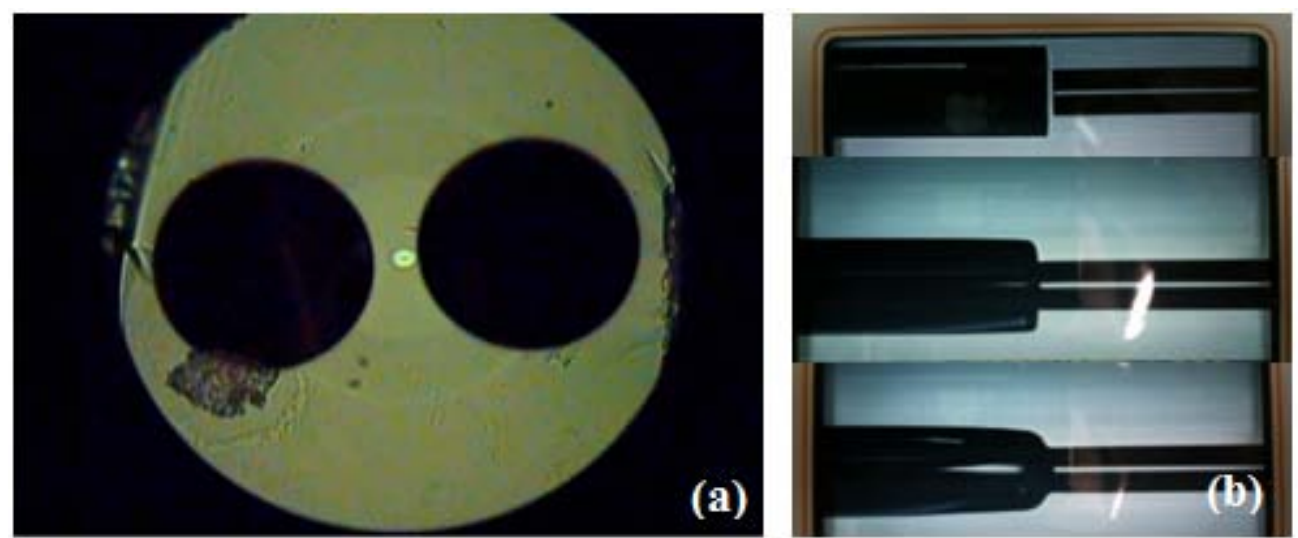

Fig. 1 (a)the microscope cross-section image of the twin-air-hole fiber with elliptical off-center core; (b) the fusion splice and hole sealing of the large diameter twin-air-hole fiber with Corning SMF-28 fiber.

The spliced fiber was loaded in a hydrogen chamber at 2400-psi and room temperature for 2 weeks before UV writing. 3-cm long seeding FBG with Bragg wavelength at 1545-nm was inscribed into the fiber core with 248-nm KrF excimer laser and 1070-nm pitch phase mask. Due to the large cladding diameter, rapid escape of loaded hydrogen from the fiber core to the air holes, and UV light diffraction in the air holes, it is difficult to write strong FBGs in the twin-air-hole fiber. Excessive UV exposures were applied in the inscription process to obtain strong AC index contrast, which inevitably induced $>10^{-3}$ DC index change and shifted the grating peaks more than 1.5-nm to longer wavelength during the UV radiation. With $\sim 22500 \mathrm{UV}$ exposures at $\sim 50 \mathrm{~mJ} / \mathrm{cm}^{2}$ radiation intensity, relatively strong FBGs can be inscribed into the twin-air-hole fiber. The transmission and reflection spectra of the inscribed FBG are shown in figure 2a. The resonant transmission dip is more than $30 \mathrm{~dB}$ deep, and reflection peak is $15 \mathrm{~dB}$ high with flat-top spectral shape and $>1-\mathrm{nm}$ spectral width. The evolution of seeding FBG during UV writing is shown in figure 2b. The reflective peak intensity saturated after 6 minutes of radiation, while the peak wavelength continued shifting to the longer wavelength, and the peak width continued broadening. No blueshift was observed during 15 minutes of UV radiation ( 22500 exposures at $25 \mathrm{~Hz}$ laser repitition rate), which confirmed that the grating is still type-I. The over-saturated UV exposure of seeding gratings proved to be critical for the regeneration process in high temperature annealing. No regeneration was observed with 6 minutes of UV radiation ( 9000 exposures).

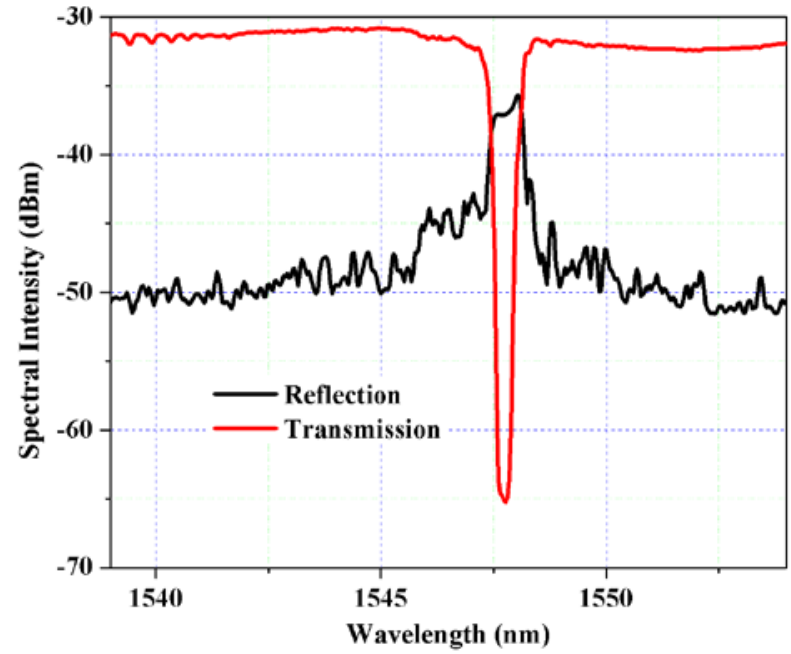

(a)

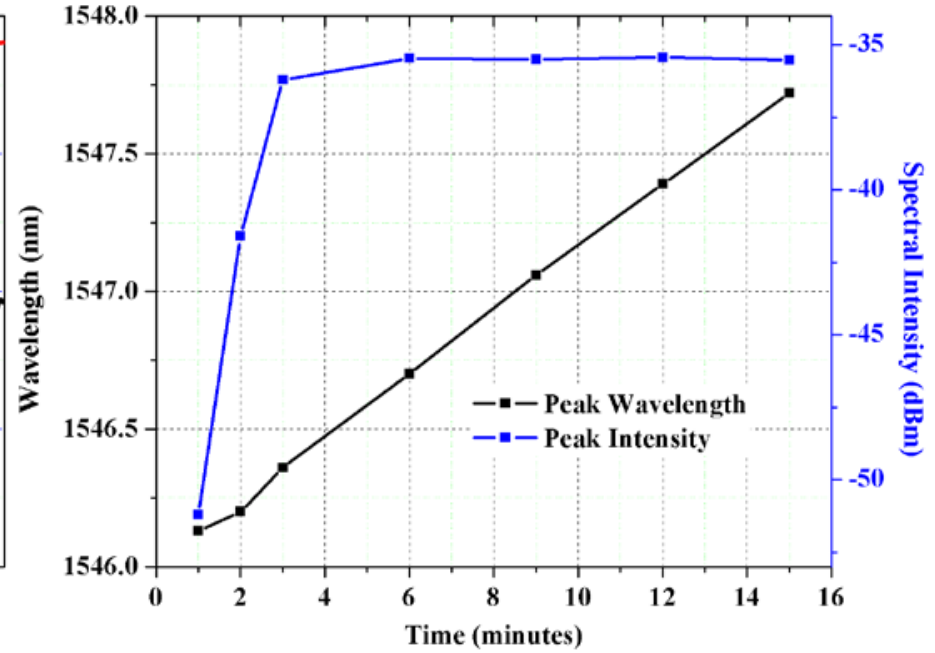

(b)

Fig. 2, (a)The transmission and reflection spectra of seeding FBG in twin-hole fiber; (b) The peak wavelength and intensity evolution of seeding FBG during 15 minutes of $25 \mathrm{~Hz} 248-\mathrm{nm}$ UV radiation. 
The fiber grating is then put into a stainless steel tube for thermal regeneration and high temperature pressure test. The fiber tube is embedded in a PID controlled furnace that is able to change and maintain temperature from room temperature up to $850^{\circ} \mathrm{C}$ with ramp rate of $200^{\circ} \mathrm{C} / \mathrm{min}$. The fiber under test is pressure sealed at both ends with carbon ferrules. The tube can be pressurized with Nitrogen gas tank and pressure regulator. The reflective spectra of the gratings are interrogated with a broadband light source (BBS, MPB EBS-7210, 1520-1610nm) and an optical spectrum analyzer (OSA, Ando 6317B, 20-pm resolution) behind a fiber recirculator. The schematic of the experimental setup is shown in figure 3.

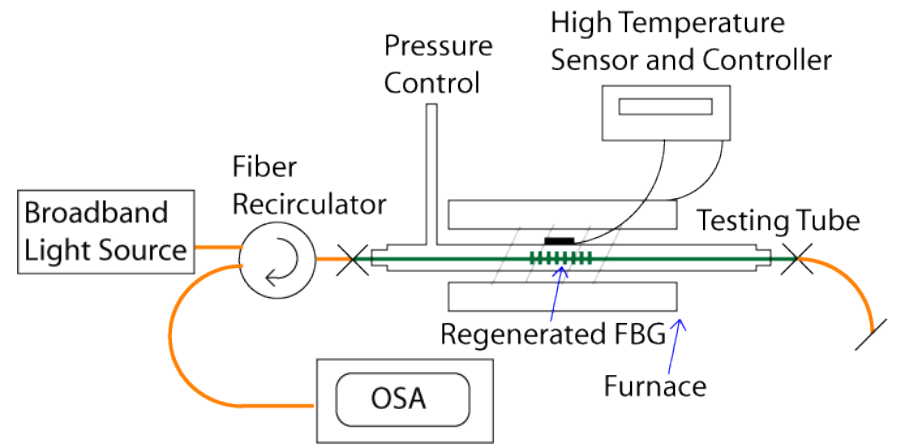

Fig. 3 the schematic of our homemade pressurized furnace for fiber grating annealing and high temperature pressure test.

The seeding FBG was annealed at $800^{\circ} \mathrm{C}$ for regeneration. Maximum ramp rate of $200^{\circ} \mathrm{C} / \mathrm{min}$ was used in the temperature rise. The fiber tube temperature reached the grating erasing point of $700-750^{\circ} \mathrm{C}$ in 5 minutes, and gradually stabilized to $800^{\circ} \mathrm{C}$ in 8 minutes. This fast ramp rate annealing recipe is different from previous reports, in which the slow ramp rate and $\sim 1$ hour pre-annealing is applied to on single-mode fiber regeneration and benefits the regenerated grating strength [12]. When the slow ramp rate recipe was applied to twin-air-hole fiber grating, no regeneration was observed. We believe this is due to the relatively weaker strength of our seeding grating and lower erasing temperature. The evolution of grating spectra in the annealing process is shown in figure 4a. Blue shift in grating wavelength was observed during regeneration, which is different from previous report on conventional single-mode fiber [9-12]. This could be attributed to the gradual relaxation of UV induced DC index in high temperature. The regeneration started before the seeding grating was completely erased. Double peaks were also observed during the process, as is shown with the green curve in figure 4a. It is still not clear to us whether this peak separation is due to the co-existence of seeding and regenerating grating, or the thermal enhancement of twin-hole fiber intrinsic birefringence. Figure 4b shows the evolution of grating wavelength and strength in a 65 hours regeneration cycle at $800^{\circ} \mathrm{C}$. Five distinct regions were identified in the regeneration process. The erase of seeding was followed by the reborn of the grating in the first 4 hours, labeled as region A and B in figure 4b. After the regenerated grating reached its maximum strength, the next 15-20 hours is labeled as region $\mathrm{C}$, in which the DC index relaxation and peak blueshifting occurred. Both the grating strength and wavelength was stabilized for 40 hours in region $\mathrm{D}$. In region $\mathrm{E}$, the testing tube and fiber grating were cooled down to room temperature in less than 2 hours. After cooling down, $2.6 \mathrm{~dB}$ boost in grating strength was observed at $24^{\circ} \mathrm{C}$. 


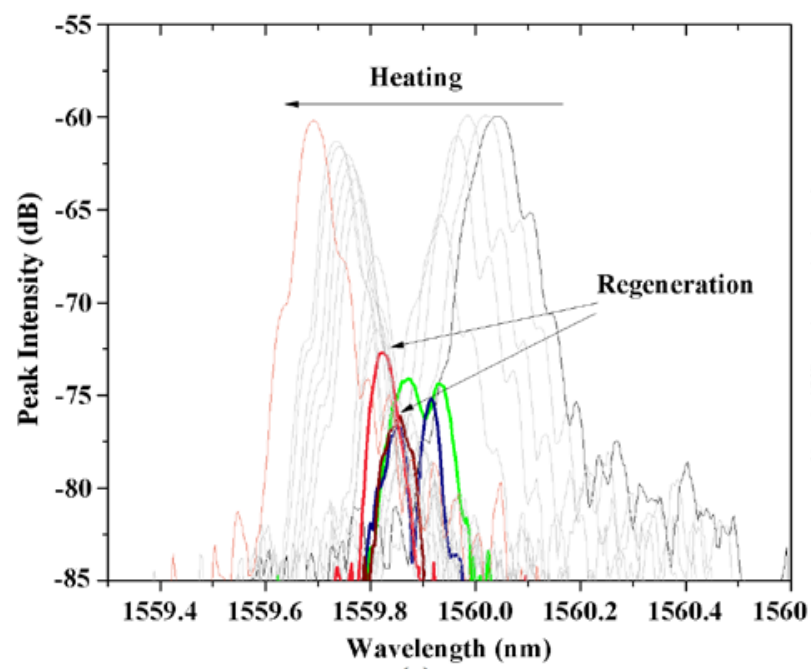

(a)

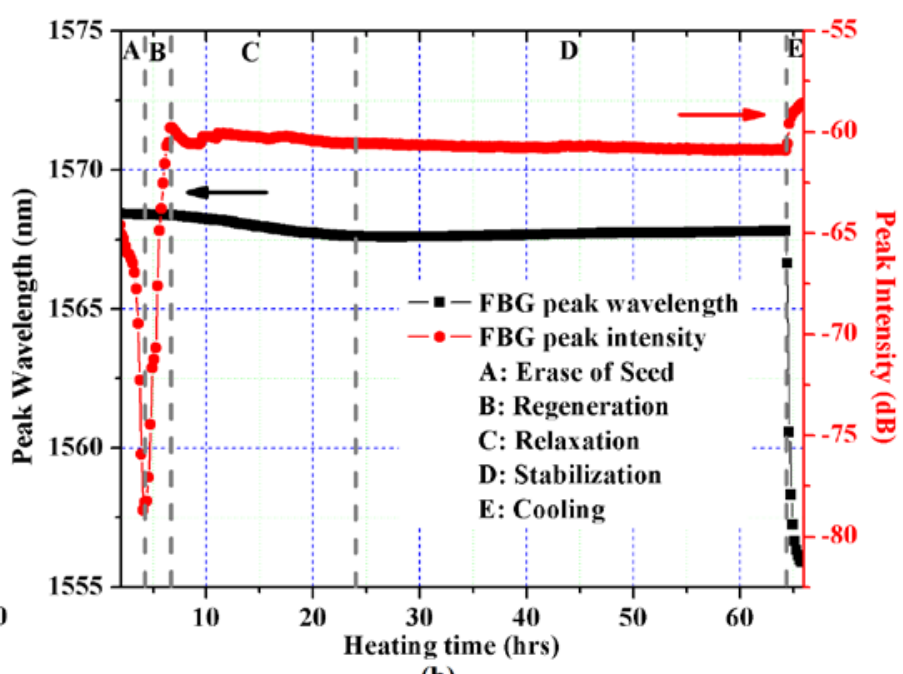

(b)

Fig. 4: Evolution of (a) grating reflection spectra, (b) grating strength and resonate wavelength during the regeneration at $800^{\circ} \mathrm{C}$.

\section{RESULTS}

The temperature and hydrostatic pressure responses of the regenerated grating in twin-hole fiber were tested in the same experimental setup. The detailed results are shown in figure 5 and 6. Figure $5 a$ shows the heating of grating at room pressure of 15-psi. Several heating and cooling cycles were carried out from $24^{\circ} \mathrm{C}$ to $800^{\circ} \mathrm{C}$ and no hysteresis was observed. In temperature range from $400-800^{\circ} \mathrm{C}$, the $\mathrm{FBG}$ peak shifts linearly as a function of temperature at a slope of $0.01518 \mathrm{~nm} /{ }^{\circ} \mathrm{C}$, which is consistent

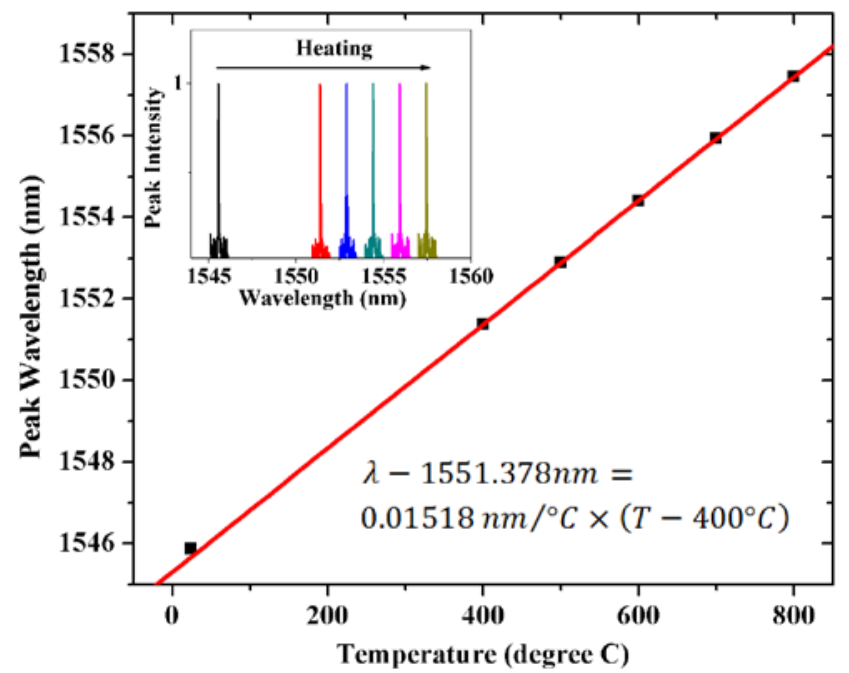

(a)

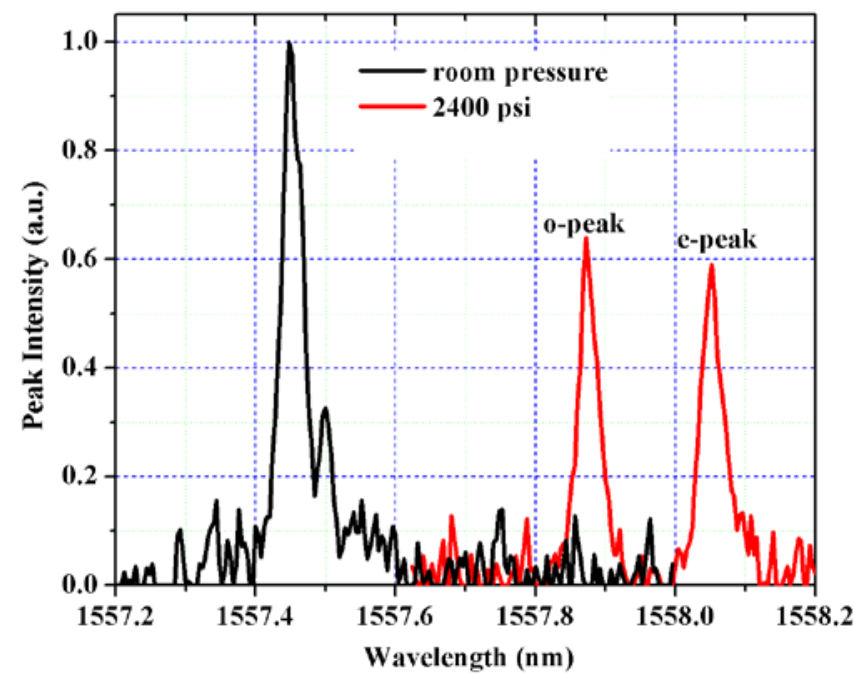

(b)

Fig. 5: The reflective spectra of the regenerated grating under pressure at 15 -psi and 2400 -psi at $800^{\circ} \mathrm{C}$.

with previous results on ultrafast laser written gratings in the same fiber [20]. The slope is slightly smaller for temperature from $24-400^{\circ} \mathrm{C}$ due to the smaller thermal-optic coefficient of silica at lower temperature. Both spectral shape and strength of the grating were not significantly changed during the heating, as shown in inset of figure 5a. At $800^{\circ} \mathrm{C}$ the regenerated grating shows a FWHM of $<40 \mathrm{pm}$ and minimal intrinsic birefringence under room pressure of $15-$ psi, as shown in figure $5 \mathrm{~b}$. When hydrostatic pressure of 2400-psi was applied, the grating shifted to longer wavelength and split into two distinct peaks 180-pm apart. The separated peaks are attributed to o and e polarizations, 
which can be confirmed by inserting a polarization beamsplitter before the OSA. This grating peak separation $\lambda_{x}-\lambda_{y}$ is generated by the birefringence in applied transverse strain $\varepsilon_{x}-\varepsilon_{y}$. And the induced birefringence is given by,

$$
\frac{\lambda_{x}-\lambda_{y}}{\lambda_{0}}=\frac{n_{e f f}^{2}}{2}\left(p_{12}-p_{11}\right)\left(\varepsilon_{x}-\varepsilon_{y}\right)
$$

Where $p_{11}$ and $p_{12}$ are strain-optic tensor component of the fiber, and $n_{\text {eff }}$ is the effective refractive index in the fiber core. Linear dependence of the birefringence upon applied hydrostatic pressure is expected. The slope is expect to vary with ambient temperature due to the temperature dependences of $n_{\text {eff }}, p_{11}$ and $p_{12}$.

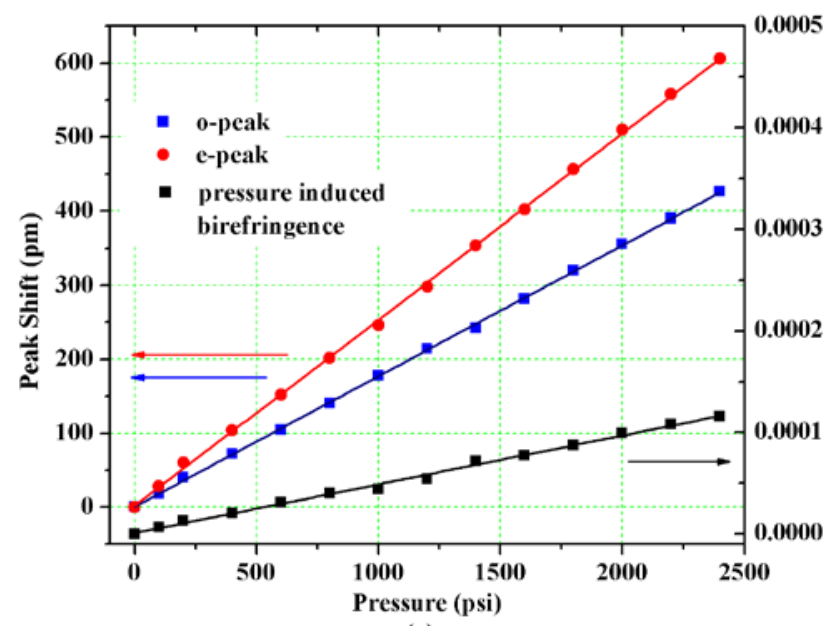

(a)

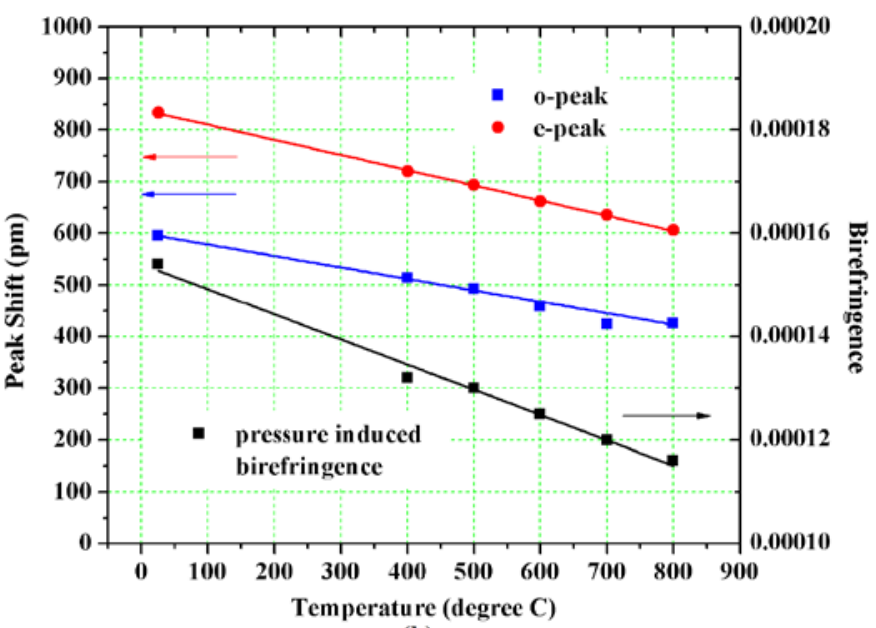

(b)

Fig. 6: The regenerated grating wavelength vs. (a)external pressure from 15 -psi to 2400 -psi at $800^{\circ} \mathrm{C}$; (b) temperature from $24^{\circ} \mathrm{C}$ to $800^{\circ} \mathrm{C}$ under external pressure of 2400 -psi.

The peak separation and induced birefringence are studied under pressure from $15-2400 \mathrm{psi}$ at $800^{\circ} \mathrm{C}$, and at temperature from $24-800^{\circ} \mathrm{C}$ under $2400 \mathrm{psi}$. The results are shown in figure $6 \mathrm{a}$ and $6 \mathrm{~b}$ respectively. The resonant wavelengths for both polarizations show linear dependences to the external pressure over the entire testing range of pressure. Thanks to the sharp grating linewidth and minimal intrinsic birefringence, the splitting between peaks can be identified as small as $100 \mathrm{psi}$. The pressure sensitivity of the grating sensor is 13.3 -psi per pm at $800^{\circ} \mathrm{C}$, which is also consistent with the results from ultrafast laser written type-II gratings [20]. Under 2400 psi, the pressure response and induced birefringence is $25 \%$ smaller at $800{ }^{\circ} \mathrm{C}$ than at $24^{\circ} \mathrm{C}$, which indicates the glass softening and decrease of strain-optic coefficients at high temperature. The temperature and pressure responses of the regenerated grating sensor can be summarized as,

$$
\begin{aligned}
& \left(\begin{array}{l}
\Delta \lambda_{o} \\
\Delta \lambda_{e}
\end{array}\right)=1.518 \times 10^{-2} \mathrm{~nm} /{ }^{\circ} \mathrm{C} \Delta T+\left(\begin{array}{l}
2.521 \times 10^{-4} \mathrm{~nm} / \mathrm{psi}-9.185 \times 10^{-8} \mathrm{~nm} /{ }^{\circ} \mathrm{C} / \mathrm{psi} \Delta T \\
3.526 \times 10^{-4} \mathrm{~nm} / \mathrm{psi}-1.232 \times 10^{-7} \mathrm{~nm} /{ }^{\circ} \mathrm{C} / \mathrm{psi} \Delta \mathrm{T}
\end{array}\right) \Delta P \\
& \Delta \lambda_{o, e}=\lambda-1551.378 \mathrm{~nm}, \Delta T=T-400^{\circ} \mathrm{C}, \Delta P=P-0 \mathrm{psi}
\end{aligned}
$$

Both temperature and pressure can be measured simultaneously according to the response matrix above. With a tunable laser of 1-pm resolution, the temperature resolution is less than $0.1^{\circ} \mathrm{C}$ from $400-800{ }^{\circ} \mathrm{C}$ and the pressure resolution is less than 1 atmosphere pressure (15-psi) from 1-160 atmosphere pressure (15-2400 psi).

\section{DISCUSSION AND SUMMARY}

The maximum pressure used to test the FBG sensor was limited by our $\mathrm{N}_{2}$ tank pressure. It is believed that air-hole silica fiber has potential to sustain much higher pressure up to 10,000-psi according to our simulation. The tested temperature of $800{ }^{\circ} \mathrm{C}$ is also limited by our homemade pressurized high temperature furnace. By further optimization of the writing of seed grating and annealing schedule, it is possible to push up the survival and operating temperature of the regenerated 
pressure sensor to higher than $1000{ }^{\circ} \mathrm{C}$ [11-12]. On the other hand, the fiber geometries can also be further optimized with FEA simulations, to achieve higher pressure sensitivity and lower temperature/pressure cross-sensitivities [18-19]. In comparison with the previous results using type-II FBG [20], better measurement sensitivity and accuracy, better multiplexing capability and the capability of temperature/pressure discrimination are achieved with the type-I regenerated FBGs in twin-hole fiber.

In summary, this paper applies the thermal regeneration technique to twin-hole microstructure fibers, and presents hightemperature stable fiber pressure sensor for industry applications. The sensor shows accurate and reliable operation from $24-800^{\circ} \mathrm{C}$ for hydrostatic pressure from $15-2400$ psi. Compared with interferometer based fiber pressure sensors, the regenerated grating sensor in air-hole microstructured fibers can be multiplexed. A high temperature and pressure sensing network can be formed with large numbers of these sensors on a single fiber feedthrough, and interrogated with a single optical sensing unit.

This work was supported by the National Science Foundation (CMMI-0644681). Kevin P. Chen's e-mail address is pec9@pitt.edu.

\section{RERERENCES}

[1] Xiao. H., Deng, J., Wang, Z., Huo, W., Zhang, P., Luo, M., Pickrell, G. R., May, R. G., and Wang, A., "Fiber optic pressure sensor with self-compensation capability for harsh environment applications”, Opt. Eng. 44, 054403 (2005).

[2] Zhu, Y., Cooper, K. L., Pickrell, G. R., and Wang, A., "High-Temperature Fiber-Tip Pressure Sensor”, J. Lightwave Technol. 24, 861 (2006).

[3] Wu, C., Fu, H.Y., Qureshi, K.K., Guan, B. and Tam, H.Y., "High-pressure and high-temperature characteristics of a Fabry-Perot interferometer based on photonic crystal fiber”, Optics Letters, 36, 412, (2011).

[4] Fokine, M., "Formation of thermally stable chemical composition gratings in optical fibers," J. Opt. Soc. Am. B, 19(8), 1759-1765 (2002).

[5] Butov, O.V., Dianov, E.M., and Golant, K.M., "Nitrogen doped silica-core fibres for Bragg grating sensors operating at elevated temperatures,” Meas. Sci. Technol, 17(5), 975-979 (2006).

[6] Aslund, M., and Canning, J., "Annealing properties of gratins written into UV-presensitized hydrogen-outdiffused optical fiber,” Optics Letters, 25(10), 692-694 (2000).

[7] Grobnic, D., Smelser, C.W., Miharlov, S.J., and Walker, R.B., "Long-term thermal stability tests at $1000^{\circ} \mathrm{C}$ of silica fibre Bragg gratings made with ultrafast laser radiation”, Mea. Sci. Technol. 17(5), 1009-1013 (2006).

[8] Groothoff, N., and Canning, J., “Enhanced type IIA gratings for high-temperature operation,” Optics Letters, 29(20), 2360-2362 (2004).

[9] Zhang, B. and Kahrizi, M., "High-temperature resistance fiber Bragg grating temperature sensor fabrication”, IEEE Sensors Journal, 7, 586, (2007).

[10] Bandyopadhyay, S., Canning, J., Stevenson, M. and Cook, K., "Ultrahigh-temperature regenerated gratings in boron-codoped germanosilicate optical fiber using 193nm”, Optics Letters, 33, 1917, (2008).

[11] Canning, J., Stevenson, M., Bandyopadhyay, S., and Cook, K., "Extreme Silica Optical Fibre Gratings," Sensors (Basel Switzerland) 8(10), 6448-6452, (2008).

[12] Bandyopadhyay, S., Canning, J., Biswas, P., Stevenson, M. and Dasgupta, K., “A study of regenerated gratings produced in germanosilicate fibers by high temperature annealing”, Optics Express, 19, 1198, (2011).

[13] Yamate, T., Ramos, R.T., Schroeder, R.J., and Udd, E., "Thermally insensitive pressure measurements up to 300 degree C using fiber Bragg gratings written onto side hole single mode fiber,” SPIE Proceddings, Vol. 4185, pp. 628-630, 2000.

[14] Xie, H. M., Dabkiewicz, P., Ulrich, R.,“Side-hole fiber for fiber-optic pressure sensing“ Opt. Lett. 11, 333-335 (1986).

[15] Kreger, S., Calvert, S., and Udd, E., "High pressure sensing using fiber Bragg gratings written in birefringent side hole fiber" in Optical Fiber Sensors Conference 2002 (Optical Society of America, 2002)

[16] Silva-Lopez, M., Li, C., MacPherson, W. N., Moore, A. J., Barton, J. S., Jones, J. D. C., Zhao, D., Zhang, L., and Bennion, I., "Differential birefringence in Bragg gratings in multicore fiber under transverse strain" Opt. Lett. 29, 22252227 (2004).

[17] Jewart, C., Chen, K. P., Mcmillen, B., Bails, M. M., Levitan, S. P., Canning, J., and Avdeev, I. V., "Sensitivity enhancement of fiber Bragg gratings to transverse stress by using microstructural fibers” Opt. Lett. 31, 2260-2262 (2006). 
[18] Jewart, C., Xu, D., Chen, K.P. and Canning, J., "Structure optimization of air-hole fibers for high-sensitivity fiber Bragg-grating pressure sensor”, SPIE 7004, 2008.

[19] Jewart, C., Quintero, S. M., Braga, A. M. B., and Chen, K. P., "Design of a highly-birefringent microstructured photonic crystal fiber for pressure monitoring, ” Optics Express, 18(25), 25657 (2010).

[20] Jewart, C, Wang, Q., Canning, J., Grobnic, D., Miharlov, S. J. and Chen, K. P., "Ultrafast femtosecond-laserinduced fiber Bragg gratings in air-hole microstructured fibers for high-temperature pressure sensing”, Optics Letters, 35, 1443, (2010). 